\title{
A Novel Innovative Sleeping Mattress Could Possibly Save Lives and the National Economy: Small but Solid Evidence from a Medical Perspective
}

\author{
Masaki Omura', Nobuhiro Suetake1, Kenichi Itao², Takuji Yamaguchi ${ }^{3}$, Ailing $\mathrm{Hu}^{3}$, \\ Hiroyuki Kobayashi1,3 \\ ${ }^{1}$ Department of Hospital Administration, Juntendo University Graduate School of Medicine, Tokyo, Japan \\ ${ }^{2}$ WINFrontier Co. Ltd., Tokyo, Japan \\ ${ }^{3}$ Center for Advanced Kampo Medicine and Clinical Research, Juntendo University Graduate School of Medicine, Tokyo, Japan \\ Email: m-omura@juntendo.ac.jp
}

How to cite this paper: Omura, M., Suetake, N., Itao, K., Yamaguchi, T., Hu, A.L. and Kobayashi, H. (2020) A Novel Innovative Sleeping Mattress Could Possibly Save Lives and the National Economy: Small but Solid Evidence from a Medical Perspective. Health, 12, 811-827.

https://doi.org/10.4236/health.2020.127059

Received: June 21, 2020

Accepted: July 17, 2020

Published: July 20, 2020

Copyright $\odot 2020$ by author(s) and Scientific Research Publishing Inc. This work is licensed under the Creative Commons Attribution International License (CC BY 4.0).

http://creativecommons.org/licenses/by/4.0/

\begin{abstract}
The RAND Corporation reported that insufficient sleep causes an economic loss of $\$ 138$ billion (2.92\% of GDP) in Japan every year. In this study, we investigated the sleep improvement effect of a novel innovative sleeping mattress called "AiR SI" (Nishikawa Co., Ltd.) to find a simple method to solve the problem of sleep deprivation. We conducted a 2-week randomized, open-label, crossover, self-controlled study in 14 healthy adults in their 30 s to 50s (control: 1 week, AiR SI: 1 week), with sleep quality as the primary endpoint as well as salivary components (cortisol, melatonin) and autonomic nervous function (sympathetic nerve, parasympathetic nerve) as secondary endpoints. Trends toward improvement in sleep were suggested for all the endpoints, regardless of differences in the subject background. The results suggested that the use of AiR SI for 1 week not only improved sleep but normalized both the endocrine and autonomic functions as well. We conclude that, by using a sleeping mattress with a high sleep improvement effect, the user may easily achieve higher labor productivity and have a lower mortality risk. Eliminating sleep deprivation with a sleeping mattress may lead to a reduction in national economic losses and ultimately produce significant economic effects.
\end{abstract}

\section{Keywords}

Sleeping Mattress, Sleep Deprivation, Mortality Risk, Autonomic Nervous System, Medical Economy 


\section{Introduction}

According to the RAND Corporation, a nonprofit global policy think tank in the U.S., an individual that sleeps on average less than 6 hours per night has a 2.4 percentage point higher productivity loss in the workplace and a $13 \%$ higher mortality risk than an individual sleeping between 7 and 9 hours per night. Insufficient sleep among their populations costs the five OECD countries under consideration (U.S., UK, Germany, Japan, Canada) up to $\$ 680$ billion of economic output every year. Among these countries, relative to the size of the overall economy, the estimated annual economic loss for Japan is the largest, which is $\$ 138$ billion (2.92\% of its GDP) [1]. In addition, the survey results reported by the Ministry of Health, Labor and Welfare show that almost half of the Japanese people in their 30s to 50s have an average sleeping time (average for the past month) of less than 6 hours, and about $30 \%$ of them are not obtaining sufficient rest by sleep, highlighting the fact that many Japanese people have insufficient sleep, especially around their 40s [2].

When calculated simply by labor productivity at the time of reporting by the RAND Corporation, if the sleep time of all people sleeping less than 6 hours was extended by one hour, an economic effect of up to $\$ 254$ billion and $\$ 52$ billion could be produced for the five OECD countries and Japan, respectively. Furthermore, the amount of economic loss is expected to increase to up to $\$ 775$ billion for the five OECD countries and \$156 billion (3.30\% of GDP) for Japan in 2030 [1]. The economic effect of sleep improvement can be enormous, taking into account the reduced mortality risk as well.

Since insufficient sleep duration has been linked with the leading causes of death including heart disease, cancer, unintentional injuries, stroke, Alzheimer disease, diabetes, influenza, pneumonia, hypertension, and suicide [3], it affects not only the health condition of the individuals but also their work efficiency. Therefore, it is expected that sufficient rest by improving sleep will lower mortality risk and improve social function and labor productivity, eventually leading to curtailment of the economic loss at the national level in the future.

Sleep improvement with a sleeping mattress can be one of the simplest methods to realize a comfortable sleeping environment, but few reports have scientifically verified it. We conducted 2-week randomized, open-label, crossover, self-controlled study for exploratory purposes to examine the effects of a sleeping mattress on sleep quality from a medical perspective, using the "AiR SI (Nishikawa Co., Ltd.)" as the test sample and the individual sleeping mattress of each subject as the control (Figure 1). The AiR SI mattress has a special structure called a three-dimensional cross-slit, and is designed to provide a comfortable sleeping environment that supports the body in a balanced manner in both postures, sideways and supine.

Sleep quality assessment (evaluating "sleep quality" with eight sleep variables) was chosen as the primary endpoint. Since sleep is closely related to stress [4], and endocrine function involved in the stress response is improved by eliminating 


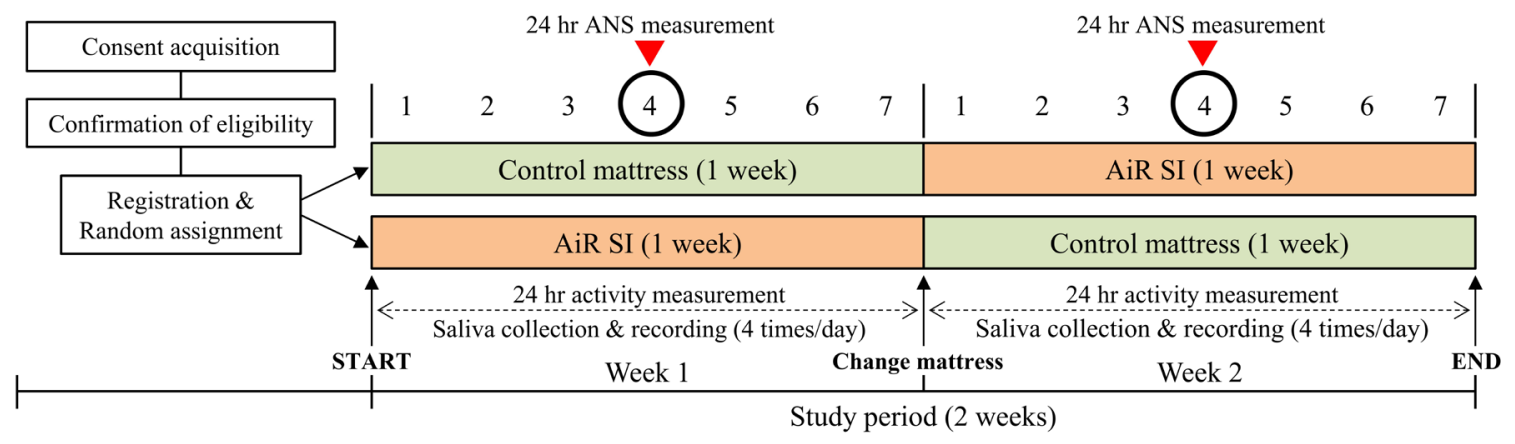

Figure 1. Example of a figure caption (figure caption). This study was a 2-week randomized, open-label, crossover, self-controlled study. The subjects were randomly assigned to one of two patterns of changing the sleeping mattress from "control to AiR SI" or "AiR SI to control." Of the 2 weeks of the study period, subjects used the control mattress for 1 week and AiR SI for another week.

sleep deprivation [5], we decided to measure physiological changes that relate to the subjects' stress response as a secondary endpoint. Two neuroendocrine systems are involved in the expression of the stress response, the hypothalamic-pituitary-adrenal cortex system (HPA axis) and the hypothalamic-sympatheticadrenal-medullary system (SAM axis) [6] [7]. Thus, salivary components (cortisol, melatonin) were included as the HPA index, and autonomic nervous (sympathetic, parasympathetic) function as the SAM index.

\section{Materials and Methods}

1) Subjects

This study was conducted in 14 healthy adults in their 30 s to 50 s from whom consent was obtained, with the approval of the ethics committee of SOUKEN (Table 1). The inclusion criteria were: 1) have not used AiR SI or similar/competitive products within the past 3 months, 2) have not changed their sleeping mattress within the past 3 months, 3) do not smoke, 4) do not drink alcohol regularly, and 5) are not taking medication. Seven men and seven women who met the criteria were randomly assigned to one of two patterns of changing the sleeping mattress after 1 week from "control to AiR SI" or "AiR SI to control." This study was conducted at each subject's home, using their own pillows and comforters. After completion of the study, all the data were tabulated for each endpoint and compared between groups by designating the week using the control mattress as the control group and the week using the AiR SI as the AiR SI group.

2) Inspection Methods

a) Experimental design

Since the experimental conditions other than the sleeping mattress could not be standardized, we instructed the subjects to avoid extreme changes in their life rhythm during the study period by waking up, having meals, taking a bath, and going to bed at the same time of day as much as possible in order to eliminate other effects on sleep, the endocrine system, and the autonomic nervous system. 
In addition, the subjects were instructed to avoid intense exercise, alcohol, caffeinated beverages, sleep supplements, and so forth, as well as not to use electronic devices such as computers and smartphones that emit blue light for long periods of time before bedtime.

b) Measurement of activity (8 sleep variables for sleep quality assessment)

The subject wore an actigraph for 24 hours except while bathing for 14 days throughout the study period, without taking it off during sleep [8]. In this study, we used the "FS-760 (ACOS CO., LTD.)", a waist-mounted actigraph, for which a sleep/wake determination coincidence rate of $88.4 \%$ with sleep polysomnography (PSG) has been reported [9]. For the sleep/wake analysis based on the activity data, we used "SleepSignAct (KISSEI COMTEC CO., LTD.)", a sleep-wake rhythm research program. In this study, we compared changes in the following eight sleep variables for sleep quality assessment: total sleep time (TST), sleep latency (SL), wake after sleep onset (WASO), wake episodes (WE), sleep efficiency (SE), average waking duration (AWD), wake episodes longer than 10 minutes (WE $\geq 10 \mathrm{~min}$ ), and posture change $(\mathrm{PC})$.

Table 1. Subject background. The participants in this study were 14 healthy adults in their 30 s to 50 s ( 7 males: $38.0 \pm 7.2$ years old, 7 females: $47.3 \pm 9.8$ years old) with consent.

\begin{tabular}{|c|c|c|c|c|}
\hline \multirow{2}{*}{ Subject No. } & \multirow{2}{*}{ Gender } & \multicolumn{3}{|c|}{ Age (years) } \\
\hline & & All subjects & Male subjects & Female subjects \\
\hline 1 & M & 37 & 37 & - \\
\hline 2 & M & 48 & 48 & - \\
\hline 3 & $\mathrm{~F}$ & 36 & - & 36 \\
\hline 4 & F & 41 & - & 41 \\
\hline 5 & M & 32 & 32 & - \\
\hline 6 & M & 30 & 30 & - \\
\hline 7 & $\mathrm{~F}$ & 53 & - & 53 \\
\hline 8 & $\mathrm{~F}$ & 57 & - & 57 \\
\hline 9 & $\mathrm{~F}$ & 35 & - & 35 \\
\hline 10 & M & 47 & 47 & - \\
\hline 11 & $\mathrm{~F}$ & 58 & - & 58 \\
\hline 12 & M & 33 & 33 & - \\
\hline 13 & $\mathrm{~F}$ & 51 & - & 51 \\
\hline 14 & M & 39 & 39 & - \\
\hline Mean \pm SD & & $42.9 \pm 10.0$ & $38.0 \pm 7.2$ & $47.3 \pm 9.8$ \\
\hline
\end{tabular}

SD: Standard deviation, M: male, F: female. 
c) Measurement of salivary components

For saliva sampling, we used "Saliva Collection Aid (Salimetrics LLC)" to collect saliva [10] [11] [12]. For 14 days during the study period, subjects collected saliva four times a day by passive drool in the predetermined time windows [morning: upon waking up, noon: 11:00-13:00 (before lunch), evening: 17:0019:00 (before meal), night: before going to bed], recording the saliva sampling time [13]. We instructed the subjects to immediately store the saliva samples in a frozen state, and retrieved them later. For analysis of the collected saliva samples, cortisol and melatonin in saliva were quantified by enzyme immunoassay (ELISA) using the "Salivary Cortisol Enzyme Immunoassay Kit (Salimetrics LLC)" and the "Salivary Melatonin Enzyme Immunoassay Kit (Salimetrics LLC) [14]". Absorbance was measured using the "Thermo Scientific Multiskan FC Absorbance Microplate Reader (Thermo Fisher Scientific K.K.)" with the absorbance filter set at $450 \mathrm{~nm}$. In order to minimize the data variation, data from two wells per subject were quantified, and the mean value of absorbance was calculated. We compared the results between the control group and the AiR SI group, focusing on changes in the salivary concentrations of cortisol in the morning and melatonin at night.

d) Analysis of the autonomic nervous system

The autonomic nervous function was measured using the ultra-compact wearable heart rate sensor "myBeat WHS-1 (UNION TOOL CO.)". The heart rate periodically fluctuates under the influence of autonomic nervous activity related to breathing (respiratory sinus arrhythmia) and circulation (Mayer wave) [15] [16]. This "spontaneous fluctuation" is heart rate variability (HRV), which is separated into low-frequency (LF) components of 0.04 to $0.15 \mathrm{~Hz}$ and highfrequency (HF) components of 0.15 to $0.4 \mathrm{~Hz}$ by frequency analysis [17] [18]. As indices in this study, the LF/HF power ratio was used for the sympathetic activity level, and the HF component for the parasympathetic activity level [19] [20] [21]. The heart rate sensor was directly attached to the skin on the left chest using an adhesive electrode. In the event of rash, itching, and so forth at the attachment site, the position of the sensor was to be shifted. Also, the subjects were instructed to remove the sensor when bathing or getting extremely wet, and to replace the electrode seal with a new one when attaching the sensor again. The autonomic nervous function was measured only for 24 hours on the 4 th day (Day 4) of the first week and the second week in this study, taking into account the possibility of adverse reactions at the sensor attachment site, the time lag until chronic stress affects the biological rhythm, the washout period for "carry-over effect", and so on. The measured data on autonomic nervous function were exported in CSV file format using “Advanced Viewer Software (WIN Frontier Co., Ltd.)". According to the saliva collection records submitted by the subjects, we selected and extracted continuous data on LF, HF, and LF/HF for several minutes before and after four respective timepoints of saliva collection (morning, noon, evening, night) from the 24-hour continuous data on Day 4 of each week using the control mattress or AiR SI, calculating the mean values at 
each timepoint by subject to compare changes in the autonomic nervous function between the control group and the AiR SI group.

3) Statistical Analyses

A one-way repeated-measures analysis of variance was performed for the data on sleep quality assessment (each of the eight sleep variables), salivary cortisol concentration in the morning, and salivary melatonin concentration at night, whereas a two-way repeated-measures analysis of variance was performed for the autonomic function data on sympathetic nerve (LF/HF) and parasympathetic nerve (HF). In both cases, Bonferroni's method was used for multiple comparisons. For statistical analysis, IBM SPSS Statistics Version 22 was used, and the significance level was set at $5 \%$.

\section{Results}

The number of valid subjects was twelve ( 5 males: $39.8 \pm 7.5$ years old, 7 females: $47.3 \pm 9.8$ years old). We adopted data from eight subjects for sleep quality assessment, excluding subjects with significant missing measurements or outliers.

1) Effect on sleep quality

Regarding sleep quality assessment, Table 2 shows the activity measurement results of both the control group and the AiR SI group on Days 1, 4, and 7, and Figure 2 illustrates the comparison between the groups for each sleep variable on Day 7.

Table 2. Measurement results of activity by sleep variable. The mean and standard deviation of the activity measurement results are shown for each group by sleep variable.

\begin{tabular}{|c|c|c|c|c|c|c|}
\hline \multirow{2}{*}{ Group } & \multicolumn{2}{|c|}{ Day 1} & \multicolumn{2}{|c|}{ Day 4} & \multicolumn{2}{|c|}{ Day 7} \\
\hline & Mean & $\mathrm{SD}$ & Mean & $\mathrm{SD}$ & Mean & $\mathrm{SD}$ \\
\hline \multicolumn{7}{|l|}{ Control } \\
\hline Total sleep time (TST) (min) & 335.8 & 56.8 & 350.8 & 61.0 & 356.5 & 71.2 \\
\hline Sleep latency $(\mathrm{SL})(\mathrm{min})$ & 6.8 & 7.8 & 9.5 & 12.7 & 9.8 & 15.6 \\
\hline Wake after sleep onset (WASO) (min) & 73.8 & 47.7 & 69.3 & 56.0 & 82.0 & 47.8 \\
\hline Wake episodes (WE) (times) & 5.0 & 3.3 & 5.4 & 3.7 & 5.6 & 2.8 \\
\hline Sleep efficiency (SE) (\%) & 79.8 & 12.7 & 81.1 & 11.9 & 78.1 & 10.4 \\
\hline Average waking duration (AWD) (min) & 13.3 & 7.0 & 10.9 & 4.8 & 14.5 & 3.6 \\
\hline Wake episodes of $\geq 10 \min (\mathrm{WE} \geq 10)$ (times) & 4.3 & 2.7 & 4.3 & 3.5 & 4.8 & 2.5 \\
\hline Posture change (PC) (times) & 23.4 & 9.1 & 22.5 & 9.6 & 24.4 & 13.7 \\
\hline Total sleep time (TST) (min) & 332.5 & 34.5 & 333.3 & 56.9 & 367.5 & 37.0 \\
\hline Sleep latency (SL) (min) & 3.5 & 3.7 & 17.5 & 16.4 & 8.0 & 12.2 \\
\hline Wake after sleep onset (WASO) (min) & 86.8 & 59.0 & 102.5 & 73.3 & 70.0 & 37.1 \\
\hline Wake episodes (WE) (times) & 5.6 & 3.4 & 5.9 & 2.7 & 4.8 & 2.3 \\
\hline Sleep efficiency (SE) (\%) & 77.8 & 12.1 & 72.2 & 13.4 & 80.6 & 9.8 \\
\hline Average waking duration (AWD) (min) & 14.7 & 4.9 & 16.9 & 7.9 & 14.3 & 4.5 \\
\hline Wake episodes of $\geq 10 \min (\mathrm{WE} \geq 10)$ (times) & 4.8 & 3.4 & 4.5 & 2.2 & 3.9 & 1.7 \\
\hline Posture change (PC) (times) & 21.4 & 8.9 & 25.8 & 11.2 & 23.1 & 15.0 \\
\hline
\end{tabular}

SD: Standard deviation. 


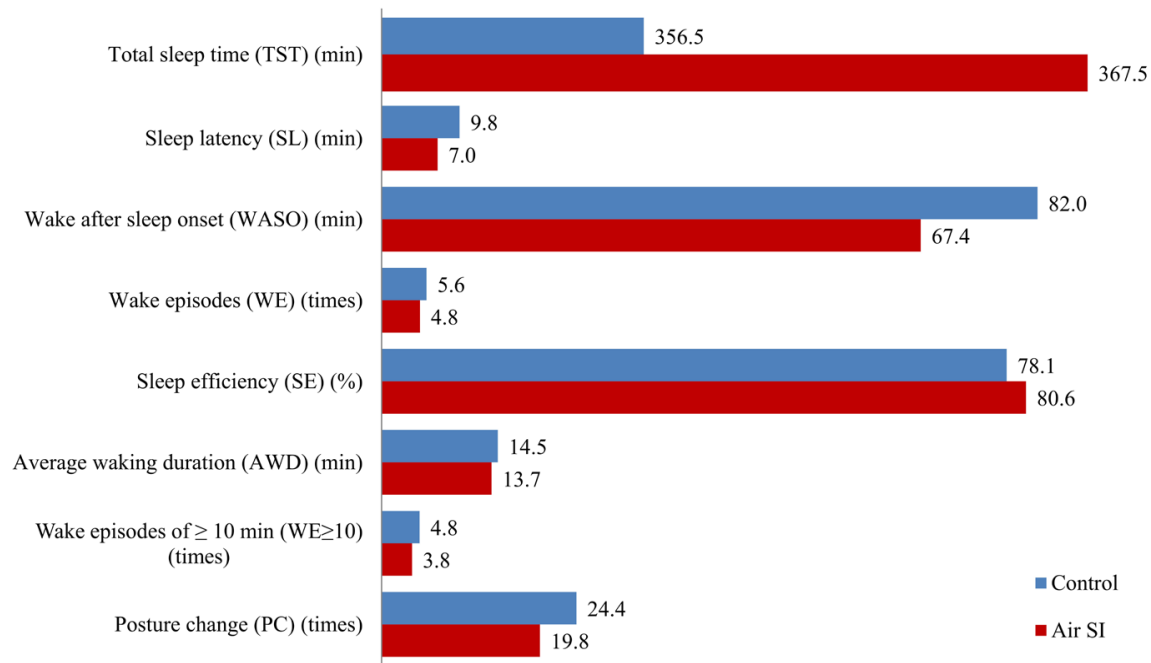

Figure 2. Comparison between groups for each sleep variable on Day 7 (control vs. AiR SI). The activity measurement results on Day 7 were compared between the groups by sleep variable. In the AiR SI group, improvements are observed in all the eight sleep variables, but there is no statistical significance for any of the variables $(\mathrm{P}<0.05)$.

Comparisons of the data between groups on Days 1 and 4 suggested a slight decrease in the "quality of sleep" in the AiR SI group for almost all the sleep variables except for "sleep latency" on Day 1. In the AiR SI group on Day 7, however, a slight trend toward improvement was observed in the "quality of sleep" for all the eight sleep variables, although there were no statistically significant differences compared to the control group for any of the variables $(\mathrm{P}=0.821$ to $1.000)$

2) Effect on salivary components

Table 3 shows the quantitative results of the salivary component concentrations in the control group and the AiR SI group, and Figure 3 and Figure 4 illustrate the changes in cortisol and melatonin levels by the group. In addition, Figure 5 and Figure 6 show a comparison of changes over time in the morning cortisol and night melatonin levels, respectively, between the groups.

The changes in salivary cortisol concentration over time were similar between the control group and the AiR SI group on Day 1. The cortisol levels were highest when waking up, and then decreased as the day progressed on Days 1, 4, and 7 in both groups (Figure 3(a) and Figure 3(b)). The cortisol levels in the morning, however, were almost unchanged in the control group over Days 1, 4, and 7, which, in contrast, gradually decreased to almost half the level of Day 1 in the AiR SI group on Day 7, with a reduced rate of $49.6 \%$. There was statistical significance between the groups on Day $7(\mathrm{P}=0.014)$, as well as within the AiR SI group between Day 1 and Day $7(\mathrm{P}=0.027)$ (Figure 5).

The morning and night salivary melatonin levels were similar between the groups on Day 1, whereas the morning level was lower and the night level was significantly higher in the AiR SI group on Day 7. None of the melatonin levels on Days 1, 4, and 7 showed a specific pattern in the control group (Figure 4(a)). 
Table 3. Quantitative results of salivary component concentration. The means and standard deviations of the salivary component concentrations (cortisol and melatonin) are shown for each group by component.

\begin{tabular}{|c|c|c|c|c|c|c|c|c|c|c|c|c|}
\hline \multirow{3}{*}{$\begin{array}{c}\text { Salivary } \\
\text { component }\end{array}$} & \multicolumn{6}{|c|}{ Control } & \multicolumn{6}{|c|}{ AiR SI } \\
\hline & \multicolumn{2}{|c|}{ Day 1} & \multicolumn{2}{|c|}{ Day 4} & \multicolumn{2}{|c|}{ Day 7} & \multicolumn{2}{|c|}{ Day 1} & \multicolumn{2}{|c|}{ Day 4} & \multicolumn{2}{|c|}{ Day 7} \\
\hline & Mean & $\mathrm{SD}$ & Mean & SD & Mean & $\mathrm{SD}$ & Mean & $\mathrm{SD}$ & Mean & SD & Mean & SD \\
\hline \multicolumn{13}{|c|}{ Cortisol (pg/ml) } \\
\hline Morning & 0.46 & 0.21 & 0.49 & 0.34 & 0.51 & 0.26 & 0.44 & 0.14 & 0.30 & 0.17 & 0.22 & 0.08 \\
\hline Noon & 0.11 & 0.08 & 0.15 & 0.15 & 0.12 & 0.08 & 0.18 & 0.16 & 0.10 & 0.05 & 0.11 & 0.05 \\
\hline Evening & 0.07 & 0.05 & 0.09 & 0.05 & 0.08 & 0.06 & 0.08 & 0.07 & 0.07 & 0.04 & 0.07 & 0.04 \\
\hline Night & 0.05 & 0.03 & 0.05 & 0.05 & 0.10 & 0.15 & 0.05 & 0.03 & 0.07 & 0.04 & 0.04 & 0.02 \\
\hline \multicolumn{13}{|c|}{ Melatonin (pg/ml) } \\
\hline Morning & 17.19 & 29.92 & 11.44 & 21.31 & 17.19 & 25.93 & 14.41 & 19.06 & 8.58 & 8.45 & 7.18 & 4.78 \\
\hline Noon & 19.45 & 30.75 & 10.96 & 17.19 & 5.95 & 11.60 & 4.37 & 3.58 & 8.35 & 14.66 & 4.88 & 3.55 \\
\hline Evening & 2.52 & 3.27 & 21.62 & 36.70 & 30.00 & 68.85 & 8.51 & 11.22 & 3.83 & 3.61 & 5.62 & 6.69 \\
\hline Night & 11.13 & 13.86 & 14.23 & 15.47 & 11.29 & 9.61 & 10.31 & 14.64 & 32.62 & 44.93 & 51.62 & 55.42 \\
\hline
\end{tabular}

SD: Standard deviation.

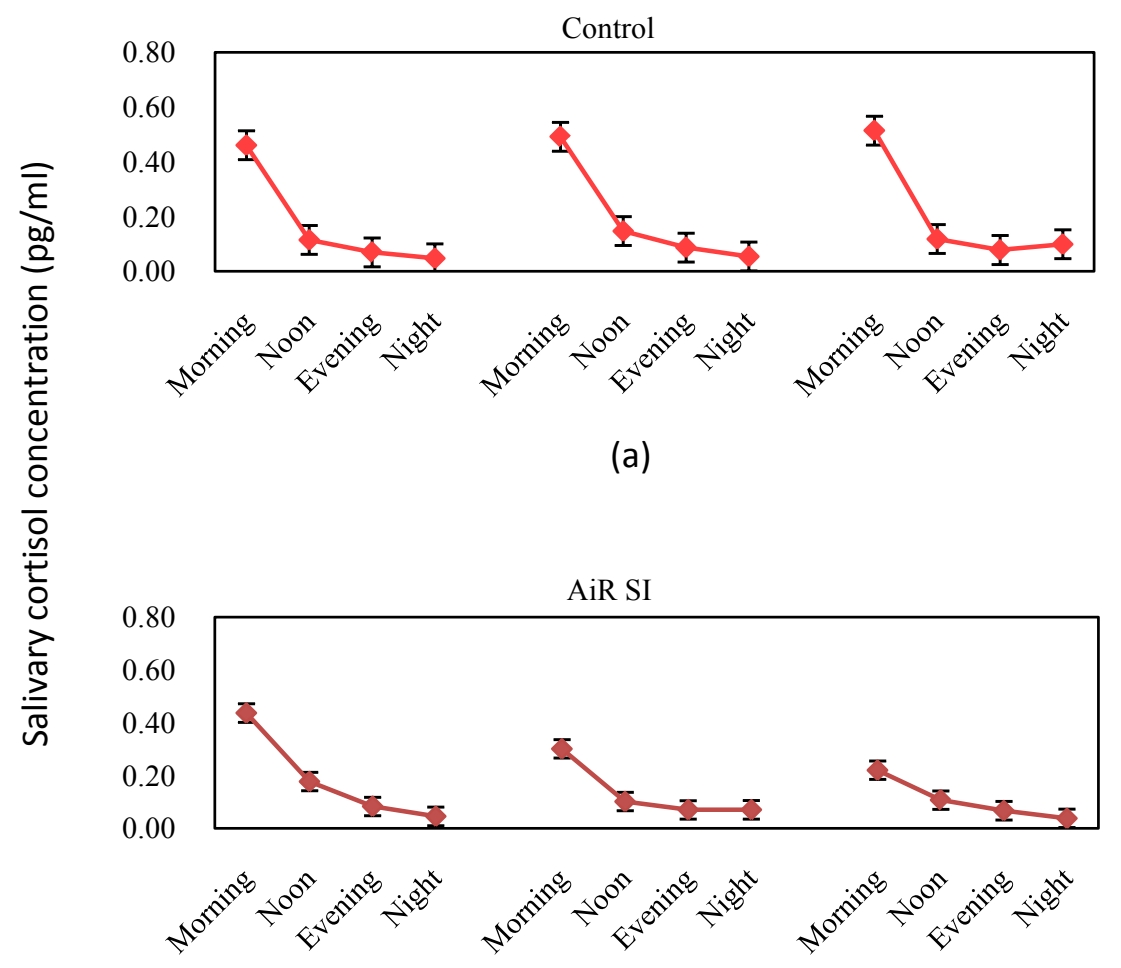

(b)

Figure 3. Change in salivary cortisol concentration. Changes in salivary cortisol concentration are shown for each group. The error bars represent standard error. 


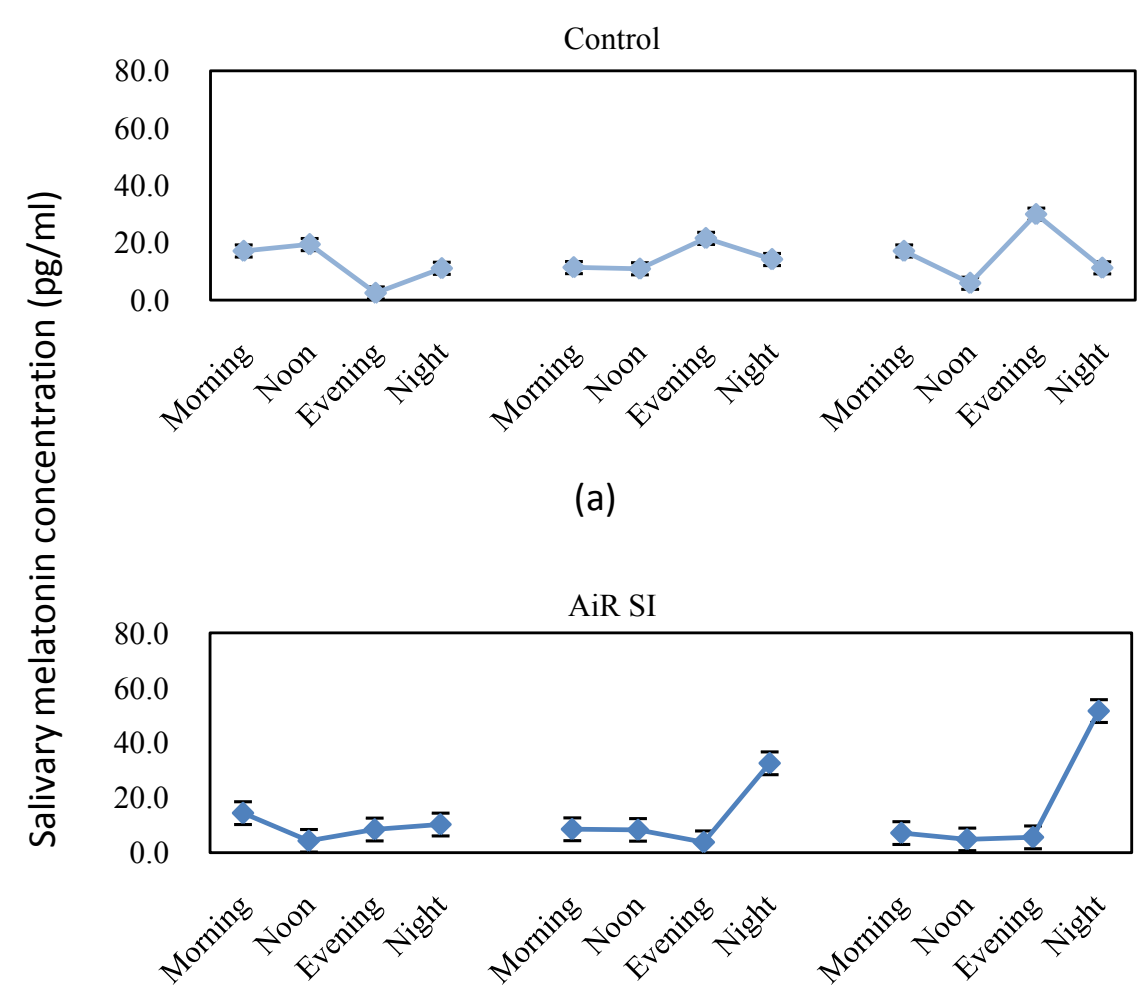

(b)

Figure 4. Change in salivary melatonin concentration. Changes in salivary melatonin concentration are shown for each group. The error bars represent standard error.

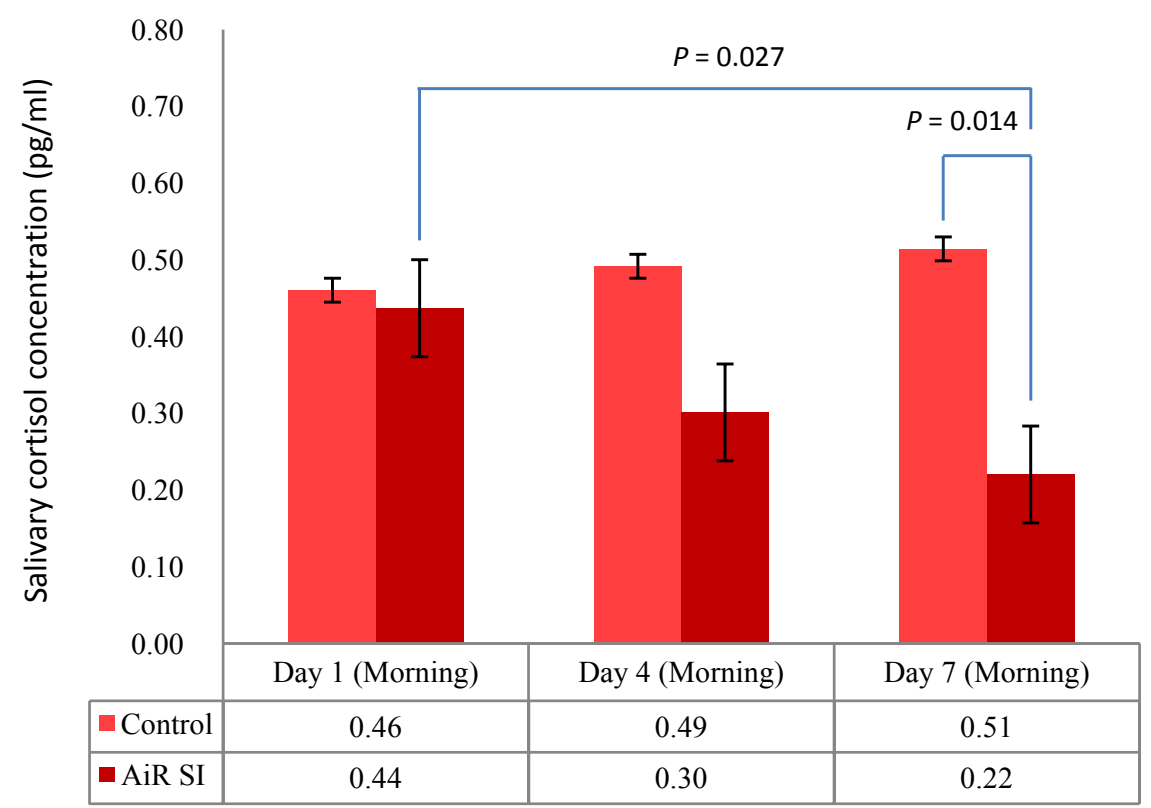

Figure 5. Comparison of salivary cortisol concentration in the morning (Control vs. AiR SI). The morning cortisol levels are compared between the groups. The error bars represent standard error. Significance probabilities are displayed for timepoints where statistical significance is observed $(\mathrm{P}<0.05)$. 


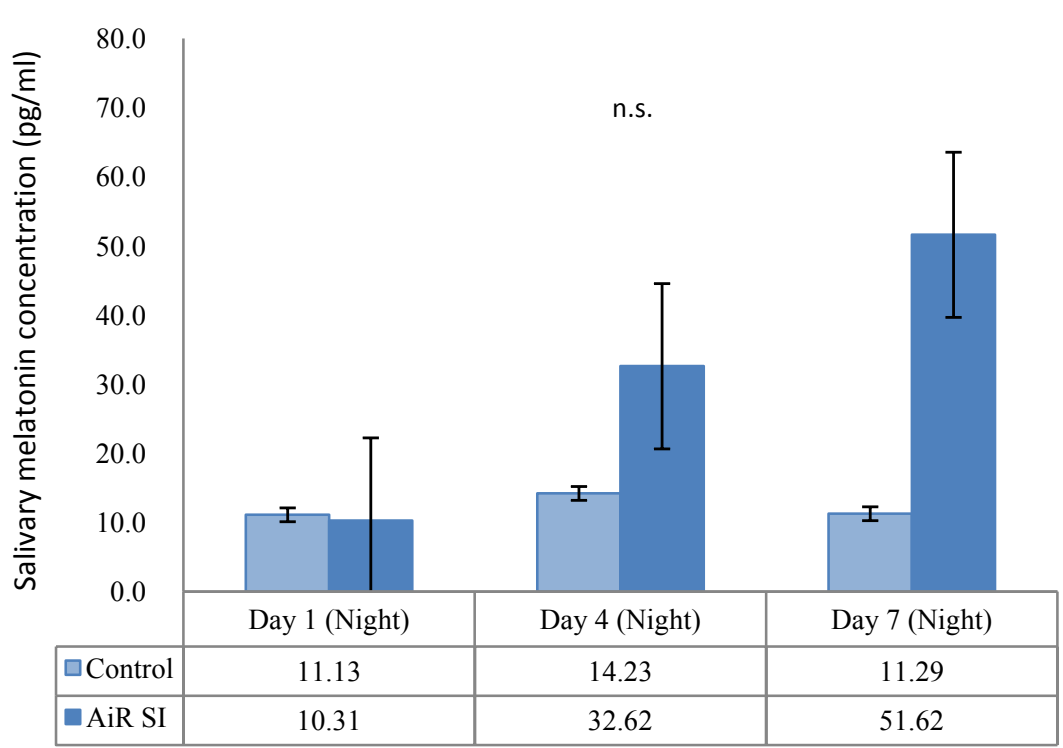

Figure 6. Comparison of salivary melatonin concentration at night (Control vs. AiR SI). The night melatonin levels are compared between the groups. The error bars represent standard error. There is no statistical significance between any timepoints $(\mathrm{P}<0.05)$.

On the other hand, they were low from morning through daytime, and increased rapidly from the evening toward the night, showing a specific pattern in the AiR SI group on Days 4 and 7 (Figure 4(b)). The night melatonin levels were almost unchanged over Days 1, 4, and 7 in the control group, whereas they gradually increased from Day 1 and peaked on Day 7 in the AiR SI group. Comparing Days 1 and 7 in the AiR SI group, the melatonin concentration at night increased five-fold (500.8\%), but no statistical significance was observed ( $P=$ 0.311) (Figure 6).

3) Effect on the autonomic nervous function

Table 4 shows the measurement results of autonomic nervous function in the control group and the AiR SI group on Day 4. Figure 7 and Figure 8 illustrate changes in the activity levels of the sympathetic nerve (LF/HF) and the parasympathetic nerve (HF) by group, and Figure 9 and Figure 10 compare the respective activity levels at the four measurement time points (morning, noon, evening, night) between the groups.

In the control group, the sympathetic activity level was high from night (5.97) till morning (8.78), and low in the afternoon from noon (3.27) to evening (3.64), with the highest parasympathetic activity level in the evening $\left(14.64 \mathrm{~ms}^{2}\right.$ ) (Figure 7(a) and Figure 8(a)). On the other hand, in the AiR SI group, the sympathetic activity level increased from morning (2.63) to noon (8.11), and decreased from evening (4.71) to night-time (2.06) after peaking around noon, whereas the parasympathetic activity level was low during the daytime from noon $\left(4.99 \mathrm{~ms}^{2}\right)$ through the evening $\left(5.95 \mathrm{~ms}^{2}\right)$ and gradually increased to peak at night $\left(8.96 \mathrm{~ms}^{2}\right)$. The HF value was higher in the AiR SI group $\left(8.96 \mathrm{~ms}^{2}\right)$ before bedtime, compared to the control group $\left(7.21 \mathrm{~ms}^{2}\right)$. 
Table 4. Measurement results of autonomic nervous function. The means and standard deviations of the measurement results of autonomic nervous function on Day 4 are shown for each group by parameter.

\begin{tabular}{|c|c|c|c|c|c|}
\hline \multirow{3}{*}{$\begin{array}{l}\text { Autonomic } \\
\text { function } \\
\text { parameter }\end{array}$} & & \multicolumn{4}{|c|}{ Day 4} \\
\hline & & \multicolumn{2}{|c|}{ Control } & \multicolumn{2}{|c|}{ AiR SI } \\
\hline & & Mean & SD & Mean & SD \\
\hline \multirow{4}{*}{$\begin{array}{l}\text { Sympathetic } \\
\text { nerve } \\
(\mathrm{LF})\left(\mathrm{ms}^{2}\right)\end{array}$} & Morning & 42.02 & 58.10 & 12.85 & 11.39 \\
\hline & Noon & 29.10 & 41.08 & 29.39 & 49.41 \\
\hline & Evening & 60.10 & 99.72 & 27.69 & 53.78 \\
\hline & Night & 51.02 & 94.64 & 15.77 & 25.98 \\
\hline \multirow{4}{*}{$\begin{array}{l}\text { Parasympathetic } \\
\text { nerve }(\mathrm{HF})\left(\mathrm{ms}^{2}\right)\end{array}$} & Morning & 7.58 & 10.07 & 6.41 & 5.73 \\
\hline & Noon & 8.83 & 8.85 & 4.99 & 6.69 \\
\hline & Evening & 14.64 & 21.44 & 5.95 & 10.02 \\
\hline & Night & 7.21 & 8.49 & 8.96 & 12.60 \\
\hline \multirow{4}{*}{$\begin{array}{c}\text { Sympathetic } \\
\text { activity level } \\
\text { (LF/HF) }\end{array}$} & Morning & 8.78 & 15.70 & 2.63 & 2.14 \\
\hline & Noon & 3.27 & 1.98 & 8.11 & 5.27 \\
\hline & Evening & 3.64 & 2.23 & 4.71 & 2.48 \\
\hline & Night & 5.97 & 4.95 & 2.06 & 2.63 \\
\hline
\end{tabular}

SD: Standard deviation.

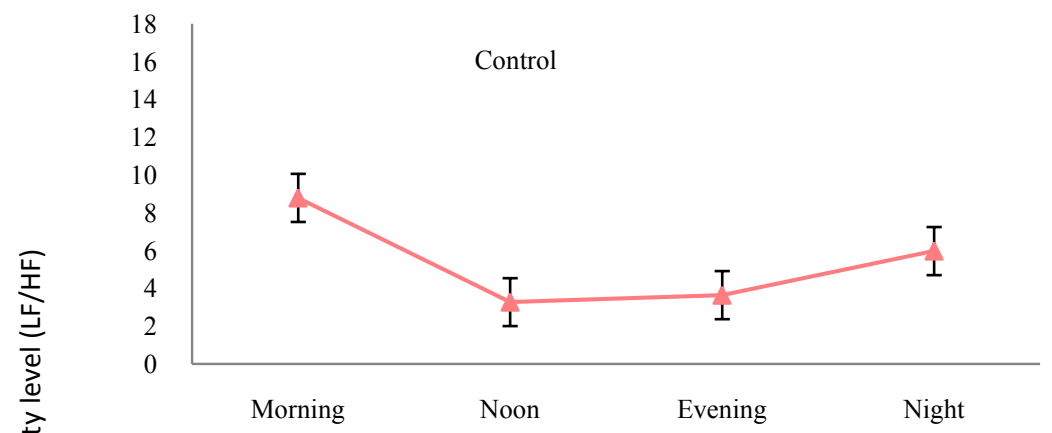

(a)

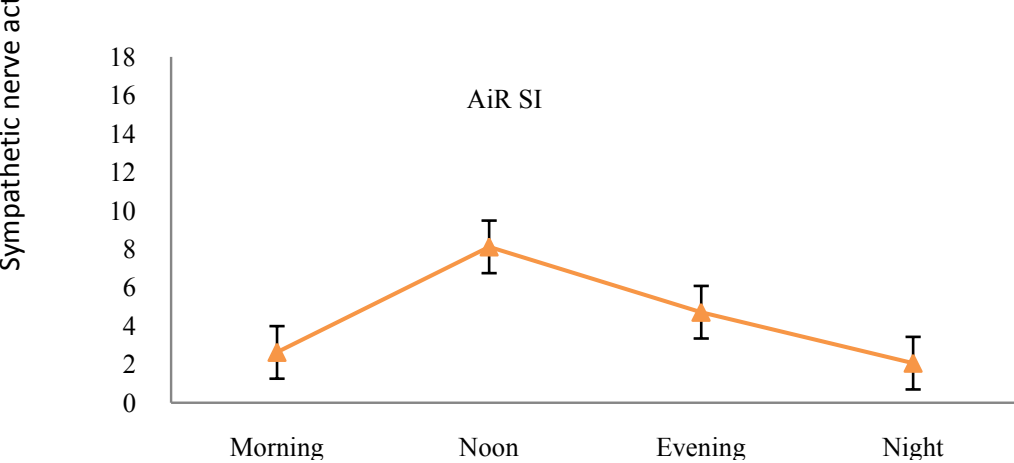

(b)

Figure 7. Change in the sympathetic activity level on Day 4. Changes in the sympathetic activity level (LF/HF) on Day 4 are shown for each group. The error bars represent standard error. 
For the sympathetic activity levels (LH/HF), there was statistical significance between the corresponding timepoints at noon $(\mathrm{P}=0.014)$ and at night $(\mathrm{P}=$ 0.042) when comparing the control and AiR SI groups. Furthermore, statistical significance was observed between the morning and noon $(\mathrm{P}=0.007)$ within the AiR SI group (Figure 9). As for the parasympathetic activity level (HF), there was no statistical significance between any of the corresponding timepoints when comparing the groups $(\mathrm{P}=0.253$ to 0.747$)$ (Figure 10).

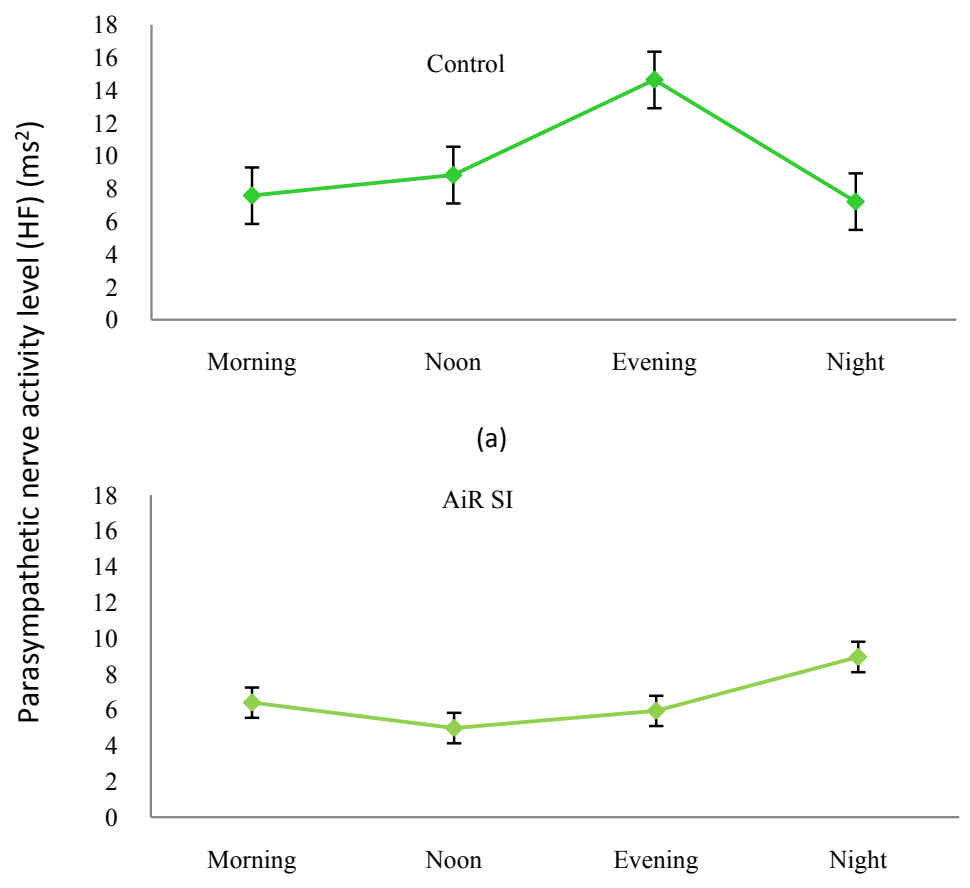

(b)

Figure 8. Change in the parasympathetic activity level on Day 4. Changes in the parasympathetic activity level (HF) on Day 4 are shown for each group. The error bars represent standard error.

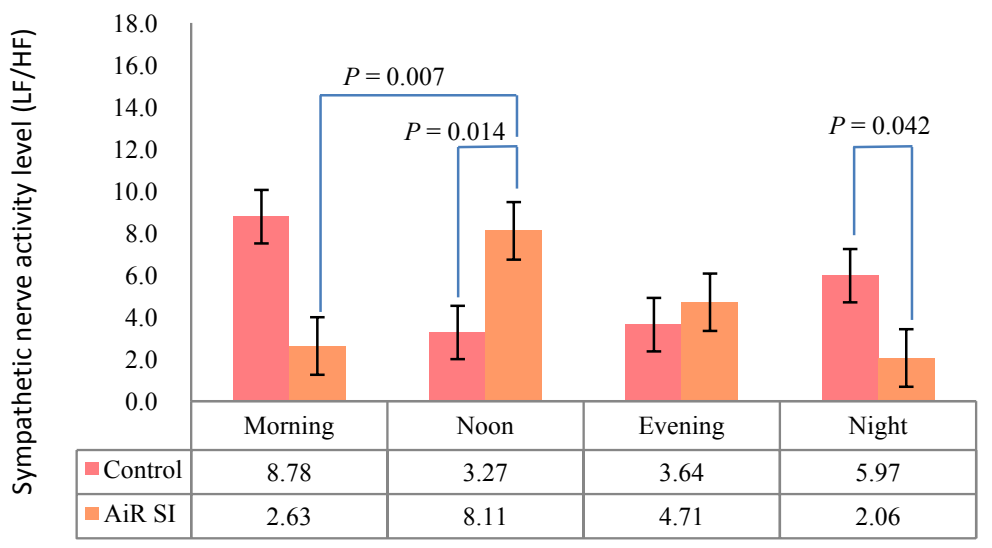

Figure 9. Comparison of the sympathetic activity level (Control vs. AiR SI). The sympathetic activity levels (LF/HF) are compared between the groups. The error bars represent standard error. Significance probabilities are displayed for timepoints where statistical significance is observed $(\mathrm{P}<0.05)$. 


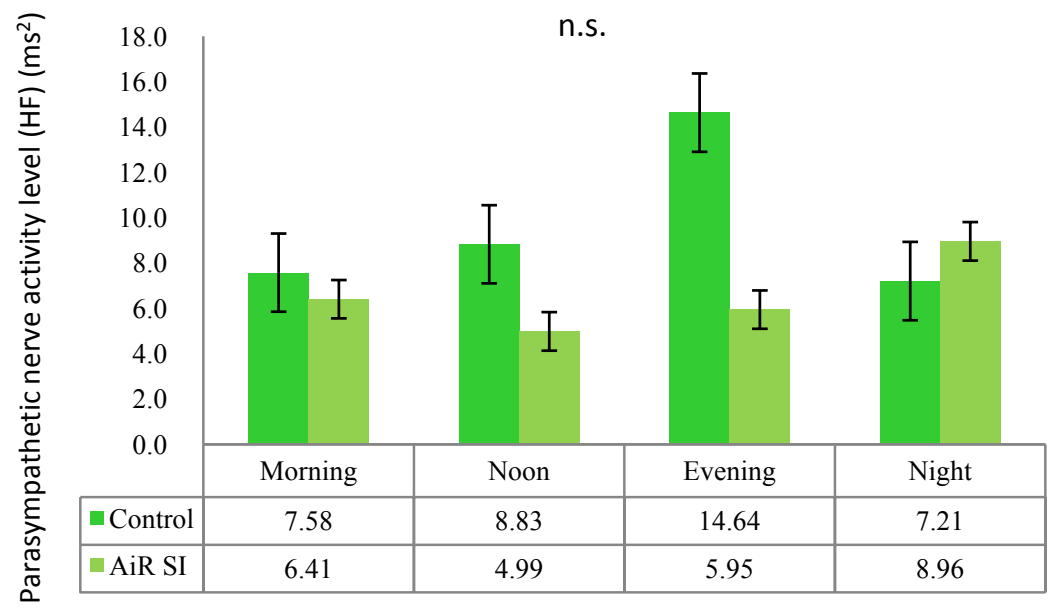

Figure 10. Comparison of the parasympathetic activity level (Control vs AiR SI). The parasympathetic activity levels (HF) are compared between the groups. The error bars represent standard error. There is no statistical significance between any timepoints $(\mathrm{P}<0.05)$.

\section{Discussion}

It has been more than a decade since the concept of sleep debt was introduced [22], and nowadays lack of sleep is a serious problem worldwide [1]. We conducted an exploratory study to evaluate the sleep improvement effect of a sleeping mattress in healthy adult men and women in their 30s to 50s using the AiR SI (Nishikawa Co., Ltd.). Among the secondary endpoints, statistically, significant improvements were shown for morning salivary cortisol concentration and sympathetic activity level. In addition, although there was no statistical significance, trends toward improvement in sleep, induction of sleepiness, and reduction in fatigue before bedtime were respectively suggested for the primary endpoint (sleep quality assessment) and the rest of the secondary endpoints (salivary melatonin concentration at night and parasympathetic activity level).

The decrease in "sleep quality" on Days 1 and 4 is considered to be a temporal and transient effect of changing sleeping mattress because improvements were observed for all the eight sleep variables in the AiR SI group on Day 7.

The salivary cortisol levels showed patterns along with the normal circadian rhythm in both groups. The morning cortisol levels, however, decreased by almost half in the Air SI group, probably due to a reduction in chronic stress in the subjects over time. The concentration of cortisol increases transiently by activation of the HPA axis with acute psychological or physical stress. The cortisol level is normally high in the morning and low from daytime to night-time due to a phenomenon known as the cortisol awakening response (CAR) [23] [24], in which the amount of cortisol secretion increases rapidly upon waking up in the morning, and it has been reported that those who are exposed to chronic or long-term stress have a higher rate of this increase [25].

It is highly likely that sleepiness was induced before bedtime in the AiR SI 
group because the melatonin secretion at night increased five-fold in 1 week in the group. Melatonin is a sleep-inducing hormone secreted from the pineal gland. It is particularly related to non-REM sleep, and its synthesis and secretion are known to be high at night and low during the daytime [26]. The salivary melatonin level showed patterns along with the normal circadian rhythm from Day 4 and thereafter in the AiR SI group, suggesting that the endocrine function may have been normalized. Cortisol and melatonin play an important role in maintaining the autonomic rhythm, and it has been noted that knowing their secretory rhythm can be an indicator of the circadian rhythm of the autonomic nervous system [11] [27] [28]. Thus, the measurement results of salivary components in this study suggest the possibility that the autonomic nervous rhythm was normalized.

Regarding autonomic nervous function, the LF/HF values in the morning and at night as well as the HF values before bedtime are considered to indicate fatigue reduction and sleep improvement in the AiR SI group. As the degree of fatigue increases, the LF/HF value of the sympathetic activity level tends to increase, while the HF value of the parasympathetic activity level tends to decrease [29]. The LF/HF value is less than 2.0 in a very resting state [30], and a decrease in "sleep quality" has been reported when it is 5.0 or more [31]. The autonomic rhythm was disturbed in the control group, whereas it showed a pattern along with the normal circadian rhythm in the AiR SI group. The results of this study not only confirm the normalization of autonomic rhythm by the use of AiR SI but also indicate the possibility of improved autonomic balance by suppressing excessive sympathetic activation and activating parasympathetic nerves.

The total sleep time (TST) on Day 7 in the control group and the AiR SI group was 5 hours 57 minutes and 6 hours 8 minutes, respectively, which was not a sufficient sleep time in either group. However, compared to the control group, the AiR SI group showed not only a tendency toward sleep improvement but also normalization in both autonomic and endocrine functions, which seemed to be due to reduction in stress and fatigue. By using the AiR SI for a week, the subjects appeared to be more active during the day, and resting and relaxed from bedtime to the morning. Therefore, it is assumed that use of the AiR SI has led to an improvement in "sleep quality" even though the improvement in total sleep time was slight.

In this study, although there were favorable tendencies in the AiR SI group when comparing the mean values between the groups, statistically significant results were not obtained with respect to sleep quality assessment, salivary melatonin concentration, or parasympathetic activity level. We believe these were mainly due to variation in the subject background. Consequently, in the next phase, we should conduct a longer-term study with a stricter design and further standardization of conditions in a larger number of subjects who have been narrowed down by thoroughly examining their background.

If it is demonstrated that the required length of sleep with sufficient quality 
can be secured by using the AiR SI, and its use spreads mainly in the population group with insufficient sleep, we can expect higher labor productivity and lower mortality risk in the users, with a possibility that this will ultimately lead to the curtailment of economic losses at the national level.

\section{Conclusion}

From the viewpoint of stress reduction and fatigue recovery of the users, the sleeping mattress can be an effective solution for alleviating sleep deprivation, a serious social problem worldwide, and this theme deserves further research in the future.

\section{Conflicts of Interest}

The authors declare no conflicts of interest regarding the publication of this paper.

\section{References}

[1] Hafner, M., Stepanek, M., Taylor, J., Troxel, W.M. and Van Stolk, C. (2017) Why Sleep Matters-The Economic Costs of Insufficient Sleep: A Cross-Country Comparative Analysis. RAND Corporation, Santa Monica, California. https://doi.org/10.7249/RB9962

[2] The Ministry of Health, Labour and Welfare (2018) The National Health and Nutrition Survey in Japan, 2017.

[3] Kochanek, K.D., Murphy, S., Xu, J.Q. and Arias, E. (2017) Mortality in the United States, 2016. NCHS Data Brief, 293, 1-8.

[4] Bassett, S.M., Lupis, S.B., Gianferante, D., Rohleder, N. and Wolf, J.M. (2015) Sleep Quality but Not Sleep Quantity Effects on Cortisol Responses to Acute Psychosocial Stress. Stress, 18, 638-644. https://doi.org/10.3109/10253890.2015.1087503

[5] Kitamura, S., Katayose, Y., Nakazaki, K., Motomura, Y., Oba, K., Katsunuma, R., Terasawa, Y., Enomoto, M., Moriguchi, Y., Hida, A. and Mishima, K. (2016) Estimating Individual Optimal Sleep Duration and Potential Sleep Debt. Scientific Reports, 6, Article No. 35812. https://doi.org/10.1038/srep35812

[6] Tanaka, Y. and Wakida, S.-I. (2010) Frontiers of Stress Research in Pharmaceutical Fields. Journal of Life Support Engineering, 22, 90-95.

[7] Cozma, S., Ghiciuc, C.M., Damian, L., Pasquali, V., Saponaro, A., Lupusoru, E.C., Patacchioli, F.R. and Dima-Cozma, L.C. (2018) Distinct Activation of The Sympathetic Adreno-Medullar System and Hypothalamus Pituitary Adrenal Axis Following the Caloric Vestibular Test in Healthy Subjects. PLoS ONE, 13, e0193963. https://doi.org/10.1371/journal.pone.0193963

[8] Kripke, D.F., Mullaney, D.J., Messin, S. and Wyborney, V.G. (1978) Wrist Actigraphic Measures of Sleep and Rhythms. Electroencephalography and Clinical Neurophysiology, 44, 674-676. https://doi.org/10.1016/0013-4694(78)90133-5

[9] Nakazaki, K., Kitamura, S., Motomura, Y., Hida, A., Kamei, Y., Miura, N. and Mishima, K. (2014) Validity of an Algorithm for Determining Sleep/Wake States Using A New Actigraph. Journal of Physiological Anthropology, 33, Article No. 31. https://doi.org/10.1186/1880-6805-33-31

[10] Kirschbaum, C. and Hellhammer, D.H. (1989) Salivary Cortisol in Psychobiological 
Research: An Overview. Neuropsychobiology, 22, 150-169. https://doi.org/10.1159/000118611

[11] Voultsios, A., Kennaway, D.J. and Dawson, D. (1997) Salivary Melatonin as a Circadian Phase Marker: Validation and Comparison to Plasma Melatonin. Journal of Biological Rhythms, 12, 457-466. https://doi.org/10.1177/074873049701200507

[12] Izawa, S., Shirotsuki, K., Sugaya, N., Ogawa, N., Suzuki, K. and Nomura, S. (2007) the Application of Saliva to an Assessment of Stress: Procedures for Collecting and Analyzing Saliva and Characteristics of Salivary Substances. Japanese Journal of Complementary and Alternative Medicine, 4, 91-101.

[13] Poll, E.M., Kreitschmann-Andermahr, I., Langejuergen, Y., Stanzel, S., Gilsbach, J.M., Gressner, A. and Yagmur, E. (2007) Saliva Collection Method Affects Predictability of Serum Cortisol. Clinica Chimica Acta, 382, 15-19. https://doi.org/10.1016/j.cca.2007.03.009

[14] Miles, A., Philbrick, D., Tidmarsh, S.F. and Shaw, D.M. (1985) Direct Radioimmunoassay of Melatonin in Saliva. Clinical Chemistry. Science, 31, 1412-1413. https://doi.org/10.1093/clinchem/31.8.1412

[15] Akselrod, S., Gordon, D., Ubel, F., Shannon, D., Berger, A. and Cohen, R. (1981) Power Spectrum Analysis of Heart Rate Fluctuation: A Quantitative Probe of Beat-to-Beat Cardiovascular Control, 213, 220-222. https://doi.org/10.21236/ADA106978

[16] Malliani, A. and Montano, N. (2002) Heart Rate Variability as a Clinical Tool. Italian Heart Journal, 3, 439-445.

[17] Malliani, A., Pagani, M., Lombardi, F. and Cerutti, S. (1991) Cardiovascular Neural Regulation Explored in the Frequency Domain. Circulation, 84, 482-492. https://doi.org/10.1161/01.CIR.84.2.482

[18] Task Force of the European Society of Cardiology and the North American Society of Pacing and Electrophysiology (1996) Heart Rate Variability: Standards of Measurement, Physiological Interpretation and Clinical Use. Circulation, 93, 1043-1065. https://doi.org/10.1161/01.CIR.93.5.1043

[19] Murakawa, Y., Inoue, H., Nozaki, A. and Sugimoto, T. (1992) Role of Sympathovagal Interaction in Diurnal Variation of QT Interval. American Journal of Cardiology, 69, 339-343. https://doi.org/10.1016/0002-9149(92)90230-V

[20] Parati, G., Saul, J.P., Di Rienzo, M. and Mancia, G. (1995) Spectral Analysis of Blood Pressure and Heart Rate Variability in Evaluating Cardiovascular Regulation. A Critical Appraisal. Hypertension, 25, 1276-1286. https://doi.org/10.1161/01.HYP.25.6.1276

[21] Thomas, B.L., Claassen, N., Becker, P. and Viljoen, M. (2019) Validity of Commonly Used Heart Rate Variability Markers of Autonomic Nervous System Function. Neuropsychobiology, 78, 14-26. https://doi.org/10.1159/000495519

[22] Dement, W.C. (2005) Sleep Extension: Getting as Much Extra Sleep as Possible. Clinics in Sports Medicine, 24, 251-268. https://doi.org/10.1016/j.csm.2004.12.014

[23] Fries, E., Dettenborn, L. and Kirschbaum, C. (2009) The Cortisol Awakening Response (CAR): Facts and Future Directions. International Journal of Psychophysiology, 72, 67-73. https://doi.org/10.1016/j.ijpsycho.2008.03.014

[24] Chida, Y. and Steptoe, A. (2009) Cortisol Awakening Response and Psychosocial Factors: A Systematic Review and Meta-Analysis. Biological Psychology, 80, 265-278. https://doi.org/10.1016/j.biopsycho.2008.10.004

[25] Izawa, S., Sugaya, N., Ogawa, N., Nagano, Y., Nakano, M., Nakase, E., Shirotsuki, 
K., Yamada, K.C., Machida, K., Kodama, M. and Nomura, S. (2007) Episodic Stress Associated with Writing a Graduation Thesis and Free Cortisol Secretion after Awakening. International Journal of Psychophysiology, 64, 141-145. https://doi.org/10.1016/j.ijpsycho.2007.01.004

[26] Higuchi, S., Motohashi, Y., Maeda, T. and Ishibashi, K. (2005) Relationship between Individual Difference in Melatonin Suppression by Light and Habitual Bedtime. Journal of Physiological Anthropology and Applied Human Science, 24, 419-423.

[27] Mcintosh, T.K., Bush, H.L., Yeston, N.S., Grasberger, R., Palter, M., Aun, F. and Egdahl, R.H. (1985) Beta-Endorphin, Cortisol and Postoperative Delirium: A Preliminary Report. Psychoneuroendocrinology, 10, 303-313. https://doi.org/10.1016/0306-4530(85)90007-1

[28] Cajochen, C., Kräuchi, K. and Wirz-Justice, A. (2003) Role of Melatonin in the Regulation of Human Circadian Rhythms and Sleep. Journal of Neuroendocrinology, 15, 432-437. https://doi.org/10.1046/j.1365-2826.2003.00989.x

[29] Kuratsune, H., Yamaguchi, K. and Sasabe, T. (2011) Evaluation of Autonomic Function in Patients with Chronic Fatigue Syndrome. Health and Labor Sciences Research Grants (Comprehensive Research Project for Persons with Disabilities) (Neural/Muscular Diseases), (Sub-Investigator) Research Year End Report.

[30] Takada, H., Takada, M. and Katayama, A. (2005) The Significance of "LF-Component and HF-Component Which Resulted from Frequency Analysis of Heart Rate" and "The Coefficient of The Heart Rate Variability"-Evaluation of Autonomic Nerve Function By Acceleration Plethysmography. Health Evaluation and Promotion, 32, 504-512.

[31] Kuratsune, H. (2011) Objective Method for Evaluating the Fatigue State of Crushing Labor. 